\title{
The Role of Software-Defined Networks for Practical Learning in the Engineering Areas ${ }^{\dagger}$
}

\author{
Cristina Alcaraz ${ }^{1, *}$, Antonio Ortega ${ }^{2}$ and Rodrigo Roman ${ }^{1}$ \\ 1 Department of Computer Science, University of Malaga, Campus de Teatinos s/n, 29071 Malaga, Spain; \\ roman@lcc.uma.es \\ 2 Radar and Electronic Defense, Indra. Carretera de Loeches 9, Torrejon de Ardoz, 28850 Madrid, Spain; \\ aofernandez@indra.es \\ * Correspondence: alcaraz@lcc.uma.es; Tel.: +34-952-139-313 \\ + Presented at the 2nd Innovative and Creative Education and Teaching International Conference \\ (ICETIC2018), Badajoz, Spain, 20-22 June 2018.
}

Published: 29 October 2018

\begin{abstract}
In this work, we have carried out a case study on the construction of a complex internetworked system that characterizes part of a Smart City within the context of Master Theses. We show that, compared to other network simulators and emulators, SDN technologies allow students to easily configure and manage large simulated communication infrastructures, and facilitate the integration of various network analysis tools.
\end{abstract}

Keywords: Software Defined Network; practical learning; virtual emulators

\section{Introduction}

In engineering fields related to industrial, telecommunication or computer sciences, it is difficult to manage the teaching and learning of the construction of complex internetworked systems. Yet the Software-Defined Network paradigm (SDN) [1] supplements many of these needs, by providing a tool where students can have direct contact with large simulated communication infrastructures, apply concepts of networks and engineering, and incorporate various types of protocols.

We have experimented with the use of Mininet and the Openflow protocol to represent SDN topologies within the context of Master Theses, so as to observe how the students adapt by themselves to the conditions of the environment to configure complex environments at a low cost and effort. In this paper we show that, apart from simplifying the deployment and configuration of software-based technologies [1], SDN helps and allows students to learn and develop the conceptual and technical lessons required to comply with the minimal competencies required.

\section{A Practical Use Case: Smart City Applications in Engineering Disciplines}

The construction and simulation of complex systems in disciplines related to the engineering is not always possible or is occasionally complicated to address. The search for specific works linked to the real world, in which a set of technologies and protocols coexist, are keys to approach the engineering students to the real world and the labor market.

Unfortunately, curricula and time restrictions limit the required time to research or prepare customizable or suitable work environments, forcing the students to concentrate all their attention, effort and time in solving specific problems, generally assuming unforeseen alternatives and/or developing the minimal goals to cover the initial expectations and overcome credits. All these handicaps, in turn, constrain or discard the exploration of other relevant factors for the learning 
process itself, such as the "research and the discovering" of suitable simulation environments according to the conditions and initial goals of the assigned work projects.

An example of these limitations is found when Smart Cities applications are sketched as part of a Master's end project. The management of smart meters in the home premise is a clear example of a Smart City application. The smart meters are devices in charge of automatically recollecting and processing the real consumption of a determined area and sending the consumption data to a concentrator. This concentrator has the ability to recollect evidence flows of several home premises and send this information to the billing entities to manage the final charging and the real demand $[2,3]$. Although the metering management process implies a significant evolution for the human being and its welfare within a society [2], numerous security and privacy breaches might arise, some of them mainly caused by the inherent vulnerabilities of the communication protocols [3-5].

In this sense, the University of Malaga (UMA) has recently led a Master's end project in order to analyze the different security weaknesses of the ModbusTCP protocol [6] in a scenario composed of eight smart meters - as illustrated in the picture to the left side of Figure 1. In this work, a man in the middle (MITM) was planned and located (as represented in the picture to the right side of Figure 1) in order to show the multiple types of cyberattacks on the path and against the confidentiality, integrity and availability of the data in the channel, among others [5]: eavesdropping, replay, data modification, false data injection, or flooding (e.g., ARP flooding, smurf or fraggle).

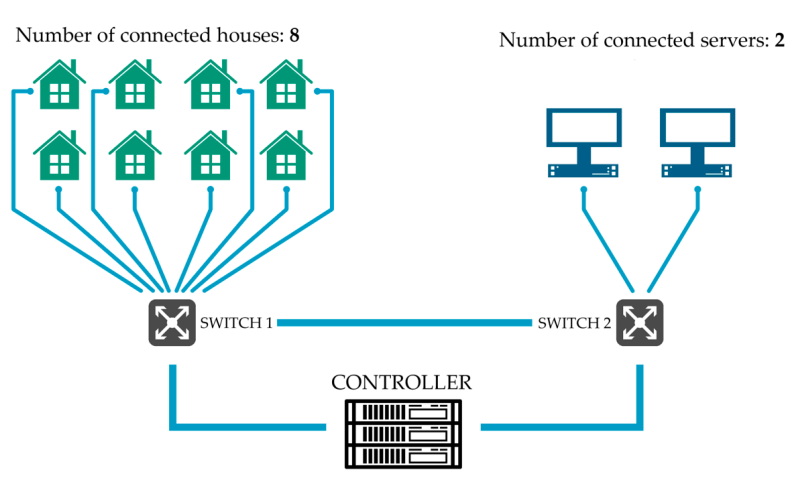

(a)

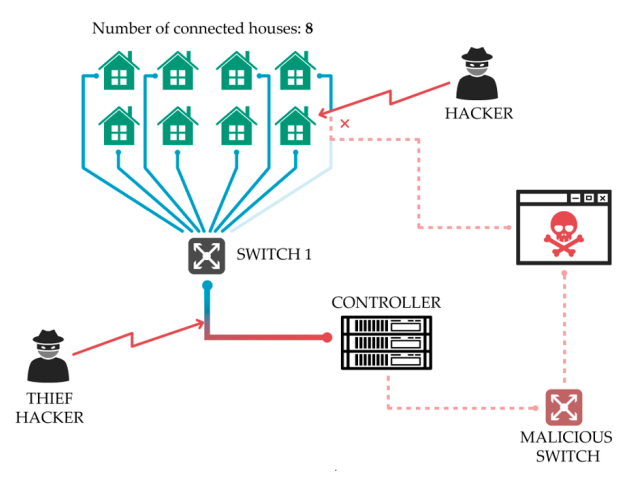

(b)

Figure 1. Use case: (a) Topological illustration of the application scenario with 8 smart meters and one controllers; (b) Representation of the practical scenario taking into account the presence of a MITM.

For the scenario construction, SDN together with Mininet [7] and OpenFlow were determinant due to the specificity of the communication protocol and the complexity of the work environment. The majority of simulators support the typical TCP/IP protocols (e.g., SSH, SSL/TLS, IPSec, etc.) but do not cover any communication protocol, and, more specifically, those related to the industry, such as ModbusTCP, DNP3 or IEC-104. In addition to this, Mininet allows for the integration of many of the hacking tools which are based on Linux distributions, facilitating the interception and manipulation tasks. Such tools include Wireshark (for dissertation of the packets and sniffing [8]), Ettercap (suite for MITM [9]), Hping3 (for flooding), Medusa (for brute force) or Scapy (packet manipulation software [10]), and are represented in Figure 2a,b.

One of the attacks that can affect these scenarios is the TCP SYN flood cyberattack. In this attack, the hacker will send a significant number of TCP packets with the SYN flag activated. This TCP traffic, generated using the tool Hping3, forces the server to start thousands of connections per second, but none of these connections finish the 3-way TCP handshake, meaning that the server will eventually run out of resources, eventually causing a critical failure. 


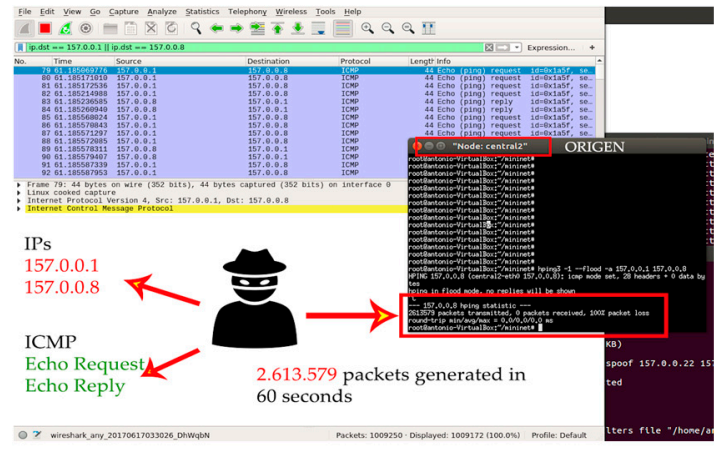

(a)

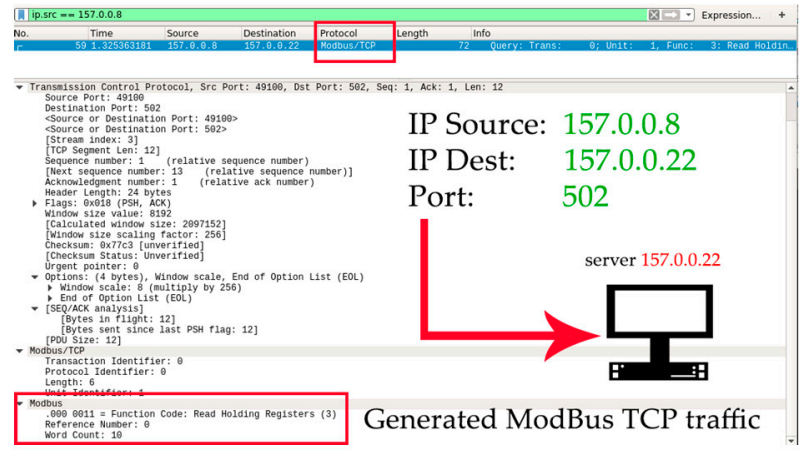

(b)

Figure 2. Cyber-attacks: (a) Representation of a denial of service using Hping3; (b) Illustration of a false injection ModbusTCP packets using Scapy.

On the other hand, false traffic can be generated using tools such as Scapy. For example, a Modbus TCP packet could be crafted (field by field) and injected into the network. These actions might allow hackers to operate different elements, just by using different TCP payloads, as well as different origin/destination IP directions.

Here, SDN offers greater capacities than simulators in general, and differs from other powerful emulators such as GNS3 by its easy network management without requiring the configuration and dependence on multiple virtual machines running as individual entities of the network. SDN offers a straightforward environment to produce simple or complex topologies and allows the student to build networks from a cheap and effective manner. Many open source routers available to be configured and included as part of GNS3, such as Quagga [11] or VyOS (compatible with Vyatta) [12], demand additional resources (more virtual space), hindering the development of practical works; whereas SDN centralizes all the network control in programmable controllers (see Figure 1 on the right side) where students can be able to interact with the own network at a low implementation cost and hardware resources.

\section{Comparison of Simulators and Emulators for Education}

As we have seen, most simulation tools (e.g., OMNet++, CISCO Packet Tracer, NS3) and emulation tools (e.g., GNS3, NetSim, CORE) could not completely replace the real environment in a learning context, as they did not have support for tools to analyse, for example, network traffic with Wireshark, interact directly with the environment, and perform cyber-attacks through specific Linux commands and applications. A summary of this analysis can be found on Table 1.

Table 1. Comparison of network simulators and emulators in a learning context.

\begin{tabular}{|c|c|c|c|c|c|c|c|}
\hline & \multicolumn{3}{|c|}{ SIMULATORS } & \multicolumn{4}{|c|}{ EMULATORS } \\
\hline & OMNeT++ & Cisco Packet Tracer 7.0 & NS3 & GNS3 & NetSim & CORE & Mininet \\
\hline Open Source & $V$ & $x$ & $\checkmark$ & $V$ & $x$ & $X$ & $V$ \\
\hline Language & $\mathrm{C}++$ & JavaScript/CSS & C++/Python & Python & Java & Python & Python \\
\hline Last Updated & 2017 & 2018 & 2018 & 2018 & 2018 & 2018 & 2017 \\
\hline $\begin{array}{l}\text { Suitable for Wireless } \\
\text { Networks }\end{array}$ & & $V$ & & $V$ & $\checkmark$ & $V$ & \\
\hline $\begin{array}{c}\text { Supports 6lowPAN traffic for } \\
\text { IoT devices }\end{array}$ & X & 4 & & & & & \\
\hline Supports SDN traffic & $X$ & X & $\checkmark$ & $V$ & $x$ & $X$ & $V$ \\
\hline Scientific Community Support & 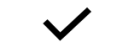 & X & $V$ & $V$ & $X$ & $V$ & $V$ \\
\hline Emulates real network interfaces & $X$ & $V$ & $X$ & $V$ & $\bar{V}$ & $\bar{V}$ & $\bar{V}$ \\
\hline User-Friendly Interface & $V$ & $V$ & $X$ & $V$ & $V$ & $V$ & $V$ \\
\hline$V X$ & +1 & -1 & +3 & +7 & +1 & +1 & +7 \\
\hline
\end{tabular}


On the other hand, Mininet, a real-time network emulator based on SDN and OpenFlow, was able to provide an easy use of the environment and configuration of software components, amongst other benefits. MiniNet allowed the dynamic creation of virtually connected networks of host, switches, links and controllers. These emulated hosts worked with Linux kernels, and as the switches support the OpenFlow protocol, it was possible to create a customizable SDN topology with a high level of flexibility. Moreover, Mininet is not limited to wired environments. A fork of Mininet, Mininet WiFi, allows users to emulate wireless networks based on WiFi technologies. Mininet WiFi provides the possibility of building complex WiFi networks using Python and it also enables the configuration of different parameters within the Wireless nodes.

\section{Conclusions and Future Work}

The main conclusions of this study focus on the beneficial characteristics of SDN as opposed to other existing technologies, and on the potential capabilities to build practical scenarios at a low infrastructural cost, configuration time and effort, in addition to favoring the students to concentrate their time in learning the essential goals of the subject-exploiting their competences properly.

Author Contributions: C.A. and A.O. conceived the study, and developed the simulated networked scenario. All authors participated in the analysis and comparison of the simulators and emulators. C.A. and A.O. wrote the paper, and R.R. edited it.

Acknowledgments: This article also has been partially supported by the "I Plan Propio Integral de Docencia, Universidad de Málaga".

Conflicts of Interest: The authors declare no conflict of interest.

\section{References}

1. Kreutz, D.; Ramos, F.M.V.; Veríssimo, P.E.; Rothenberg, C.E.; Azodolmolky, S.; Uhlig, S. Software-Defined Networking: A Comprehensive Survey. Proc. IEEE 2015, 103, 14-76.

2. Chakraborty, A.K.; Sharma, N. Advanced metering infrastructure: Technology and challenges. In Proceedings of the 2016 IEEE/PES Transmission and Distribution Conference and Exposition (T\&D), Dallas, TX, USA, 2-5 May 2016; pp. 1-5.

3. Rubio, J.E.; Alcaraz, C.; Lopez, J. Recommender system for privacy-preserving solutions in smart metering. Pervasive Mob. Comput. 2017, 41, 205-218.

4. Zeadally, S.; Pathan, A.; Alcaraz, C.; Badra, M. Towards Privacy Protection in Smart Grid, Wireless Personal Communications; Springer: Cham, Switzerland, 2012; Volume 73, pp. 23-50.

5. Alcaraz, C.: Lopez, J.; Wolthunsen, S. OCPP Protocol: Security Threats and Challenge. IEEE Trans. Smart Grid 2017, 8, 2452-2459.

6. MODBUS Application Protocol Specification V1.1b. Available online: http://www.modbus.org/docs/ Modbus_Application_Protocol_V1_1b.pdf (accessed on 1 May 2018).

7. Mininet. Available online: http://mininet.org (accessed on 1 May 2018).

8. Wireshark. Available online: https://www.wireshark.org (accessed on 1 May 2018).

9. Ettercap. Available online: https://www.ettercap-project.org (accessed on 1 May 2018).

10. Scapy. Available online: https://scapy.net (accessed on 1 May 2018).

11. Quagga. Available online: https://www.quagga.net (accessed on 1 May 2018).

12. VyOS. Available online: https://vyos.io/es/ (accessed on 1 May 2018). 